\title{
Recommendations for emergency surgery during the COVID-19 pandemic
}

Daniel Souza Lima ${ }^{1,2,3,6}$, José Alberto Dias Leite Filho ${ }^{3} \mathbb{C}$, Marcus Vinicius Silva Araújo Gurgel ${ }^{3,4} \mathbb{D}$, Antônio Fontes de Aguiar

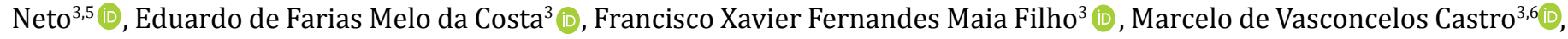
Aline Garcias Diniz ${ }^{7}$ [ , Gleydson Cesar de Oliveira Borges ${ }^{2,8}$ (D) Marcelo Augusto Fontenelle Ribeiro Junior ${ }^{9,10}$ (D)

1. Docente do Curso de Medicina da Universidade de Fortaleza (UNIFOR), Fortaleza, CE, Brasil. 2. Docente do Curso de Medicina do Centro Universitário Christus (UNICHRISTUS), Fortaleza, CE, Brasil. 3. Cirurgião do Hospital São Carlos, Fortaleza, CE, Brasil. 4. Cirurgião do Instituto do Câncer do Ceará (ICC), Fortaleza, CE, Brasil. 5. Cirurgião da Sociedade Beneficente Santa Casa da Misericórdia de Fortaleza, Fortaleza, CE, Brasil. 6. Cirurgião do Instituto Dr. José Frota (IJF), Fortaleza, CE, Brasil. 7. Discente do Curso de Medicina do Centro Universitário UNIFACISA, Campina Grande, PB, Brasil. 8. Coordenador do Serviço de Cirurgia Geral da Sociedade Beneficente Santa Casa da Misericórdia de Fortaleza, Fortaleza, CE, Brasil. 9. Cirurgião Geral do Hospital Moriah, Sorocaba, SP, Brasil. 10. Livre Docente de Cirurgia do Programa de Pós-graduação do Instituto de Assistência Médica ao Servidor Público Estadual de São Paulo (IAMSPE), São Paulo, SP, Brasil.

\section{Resumo}

\begin{abstract}
Introdução: a pandemia provocada pelo 2019 Novel Coronavírus (COVID-19) provocou um desafio global de saúde pública, com repercussões em diversos setores da sociedade. As práticas médicas necessitaram adaptar-se no atendimento ao doente portador do COVID-19 ou suspeito. Métodos: realizou-se pesquisa bibliográfica de publicações e de diretrizes de sociedades médicas em relação às abordagens cirúrgicas de emergência nesta atual pandemia. Resultados: o material bibliográfico ainda é escasso, indefinido e de baixa evidência, mas são adequados neste momento inicial de enfrentamento do COVID-19 no Brasil. Conclusão: para reduzir o risco de contaminação dos profissionais de saúde, as técnicas e decisões cirúrgicas precisam adaptar-se ao cenário de pandemia do COVID-19.
\end{abstract}

Palavras-chave: Coronavirus. Cirurgia. Emergência

\begin{abstract}
Introduction: the pandemic caused by the 2019 Novel Coronavirus (COVID-19) has caused a global public health challenge, with repercussions in several sectors of society. Medical practices needed to adapt in the care of patients with or suspected of having COVID-19. Methods: Bibliographic research of publications and guidelines of medical societies regarding emergency surgical approaches in this current pandemic was carried out. Results: the bibliographic material is still scarce, undefined and of low evidence, but which are adequate in this initial moment of coping with COVID-19 in Brazil. Conclusion: to reduce the risk of contamination of health professionals, surgical techniques and decisions need to adapt to the pandemic scenario of COVID-19.
\end{abstract}

Keywords: Coronavirus. Surgery. Emergency.

\section{INTRODUÇÃO}

Atualmente, médicos e profissionais da saúde encontram-se perante uma pandemia desafiadora causada por uma nova cepa denominada 2019 Novel Coronavírus (COVID-19). A infecção humana pelo COVID-19 ainda não tem o espectro clínico completamente descrito, bem como não se sabe com precisão o padrão de letalidade, mortalidade, infectividade e transmissibilidade. Não há vacina ou medicamento específico disponível. O tratamento é de suporte e inespecífico ${ }^{1}$. No Ceará, os números de casos confirmados laboratorialmente têm aumentado a cada dia, com a maioria dos casos concentrados na capital Fortaleza².

Os profissionais de saúde que atuam na emergência necessitam adaptar a assistência aos casos atendidos com intuito de reduzir a contaminação e a disseminação da doença. Até o início de março, a China notificou mais de 3300 profissionais de saúde infectados e, pelo menos, 22 mortes $^{3,4}$. Seguir diretrizes de proteção em procedimentos invasivos é essencial neste momento para profissionais da emergência e cirurgiões.

O objetivo deste trabalho é orientar a assistência médica para os casos de emergência cirúrgica não traumática durante a pandemia do COVID-19.

\section{MÉTODOS}

A pesquisa de artigos científicos e demais publicações foi realizada utilizando os termos "covid-19", "coronavirus", "cirurgia" na base de dados do PubMed, Lilacs/SciELO e Google, no período de 19 a 25 de março de 2020. 


\section{RESULTADOS}

A disponibilidade de publicações específicas sobre o manejo cirúrgico de pacientes confirmados com COVID-19 ou suspeitos é escassa e nova. Algumas orientações foram publicadas pela Sociedade Espanhola de Cirurgia, Colégio Americano de Cirurgiões, Sociedade Americana de Cirurgiões Gastrointestinais e Endoscopistas, Sociedade Brasileira de Atendimento Integrado ao Traumatizado, além de um único artigo com experiências médicas na China e Itália.

Com base nessas orientações, elaboram-se recomendações aplicadas ao cenário local de assistência à saúde na rede hospitalar privada, mas que também podem ser aplicadas na rede pública de saúde.

É importante salientar que essas recomendações estão sujeitas a uma revisão contínua, dependendo da situação global da pandemia e das necessidades específicas de cada hospital, bem como das recomendações das autoridades competentes e das evidências adicionais que poderão ser futuramente publicadas.

\section{DISCUSSÃO}

\section{Modo de Transmissão}

Acredita-se que a disseminação de pessoa para pessoa ocorra, principalmente, por meio de gotículas respiratórias produzidas quando uma pessoa infectada tosse ou espirra, semelhante à maneira como a influenza e outros patógenos respiratórios espalham-se. Tendo sido identificada, também, a transmissão por aerossóis em pacientes submetidos a procedimentos de vias aéreas, como a intubação orotraqueal ou aspiração de vias aéreas ${ }^{5}$.

A preocupação que o cirurgião e os demais membros da equipe cirúrgica devem ter é em relação à eliminação de aerossóis pelo doente com COVID-19 durante o peroperatório, de modo que essa preocupação determina alguns cuidados específicos e adaptações das práticas médicas.

\section{Abordagem Diagnóstica}

No atendimento de emergência, todos os casos devem ser considerados positivos para COVID-19, já que, até o momento, não temos, facilmente disponível, teste rápido de triagem. Isso vale, principalmente, para os doentes que serão submetidos a procedimentos invasivos e são instáveis clinicamente. Mantendo-se, assim, os cuidados de paramentação.

Uma possibilidade é que todo paciente suspeito de COVID-19 que precisar ser submetido a procedimento de emergência deverá, se possível, ter uma tomografia de tórax para descartar alterações compatíveis com o COVID-196.

Sintomas digestivos, especialmente diarreia (também náusea), podem preceder a clínica respiratória. Esses pacientes podem ter um prognóstico pior, pois parece estar relacionado a um aumento da carga viral e, portanto, a um aumento de complicações. Foram relatadas formas de apresentação do COVID-19 com sintomas gastrointestinais que simulam doenças cirúrgicas, principalmente de maneira muito semelhante a um quadro de pancreatite aguda, mesmo sem sintomas respiratórios ${ }^{7}$.

\section{Via de Acesso}

As doenças cirúrgicas não traumáticas mais comuns na unidade de emergência são a apendicite aguda e a colecistite aguda, que têm como tratamento padrão a cirurgia por videolaparoscopia. A realização do pneumoperitônio artificial é, possivelmente, um risco para equipe pela liberação de aerossóis. Além disso, o uso do bisturi elétrico ou ultrassônico também parece que pode levar a liberação de aerossóis, relacionado com a fumaça produzida por esses dispositivos. Estima-se que, na cirurgia laparoscópica, ocorre maior liberação de aerossóis relacionada com a fumaça do bisturi elétrico do que na cirurgia aberta ${ }^{8}$.

Há também informações relacionadas ao uso de eletrocautério e aerossolização em procedimentos gastrointestinais abertos, além de maior contato com luvas cirúrgicas que podem gerar microrrupturas nos mecanismos de barreira ${ }^{7}$.

Deve ser considerado, também, o menor tempo de internação hospitalar no pós-operatório de videolaparoscopia, em um contexto de contingência e uso excessivo de leitos hospitalares. A recomendação é que os cirurgiões avaliem a relação custo $x$ benefício do uso da abordagem laparoscópica no paciente na emergência com COVID-197,9.

\section{Cuidados com a Videolaparoscopia}

Na indicação do procedimento por abordagem via laparoscópica, alguns cuidados devem ser levados em consideração, conforme a seguir:

1. proteção individual completa deve ser rigorosamente seguida ${ }^{7,9}$;

2. entrada na sala cirúrgica após a intubação e conexão com ventilador mecânico;

3. utilização de filtro no sistema de insuflação de $\mathrm{CO}^{7,9}$;

4. realização de pneumoperitônio com agulha de veress;

5. utilização de pressões de $\mathrm{CO} 2$ menores que o habitual ${ }^{7,8}$;

6. minimização do tempo da posição de trendelenburg ${ }^{7,8}$;

7. redução do uso do eletrocautério e uso do nível mais baixo possivele $^{8}$;

8. manutenção da limpeza de sangue dos instrumentos e outros fluidos corporais durante a cirurgia, seja laparoscópica, seja via laparotomia.

9. eliminação do pneumoperitônio aspirando pelo trocater?;

10. Saída da sala cirúrgica antes da extubação do paciente.

\section{Tratamento Conservador}

O Colégio Americano de Cirurgiões propõe aos cirurgiões a 
possibilidade de priorizar o tratamento conservador para casos de apendicite aguda não complicada e complicada, iniciando antibioticoterapia intravenosa. Estratégia que poderia ser aplicada também nos casos de colecistite aguda e diverticulite aguda ${ }^{10}$.

\section{CONCLUSÕES}

No lado oposto do isolamento social, estão os profissionais de saúde na linha de frente do enfrentamento do COVID-19, com riscos maiores de infecção. A garantia de equipamentos de proteção individual deve ser priorizada, associada às adaptações da assistência e dos procedimentos cirúrgicos com intuito de reduzir o risco de infecção. É necessário elevar o nível de medidas de conscientização e proteção para o risco de exposição ocupacional em laparoscopia e cirurgia aberta tradicional. Procedimentos com exposição de aerossóis do paciente devem-se adaptar, exigindo a educação de toda equipe cirúrgica. Os profissionais de saúde são os recursos mais valiosos neste momento.

\section{REFERÊNCIAS}

1. Fortaleza. Secretaria Municipal da Saúde. Instituto Dr. José Frota. Diretriz de manejo de infecção humana pelo novo Coronavírus (SARS-CoV2) [mensagem pessoal]. Nota Técnica [Internet]; 2020 Mar [acesso 2020 Mar 19]; 1. Mensagem recebida por: <souzadl@hotmail.com

2.Ceará. Secretaria da Saúde. Doença pelo novo coronavírus (COVID-19). Inf Epidem [Internet]. 2020 Mar [acesso 2020 Mar 24]; (18): número 18: 1-9. Disponível em: https://www.saude.ce.gov.br/download/arquivos-coronaviruscovid-19/.

3. Organização das Nações Unidas. OMS alerta sobre contaminação de profissionais de saúde por Covid-19 [Internet]. ONU News; Fev 2020 [acesso 2020 Mar 19]. Disponível em: https://news.un.org/pt/story/2020/02/1704211.

4. COVID-19: protecting health-care workers. Lancet [Internet]. $2020 \mathrm{Mar}$ [acesso 2020 Mar 22]; 395(10228): 922 doi: http://dx.doi.org/10.1016/s01406736(20)30644-9. Disponível em: https://www.thelancet.com/journals/lancet/ article/PIIS0140-6736(20)30644-9/fulltext.

5. Ministério da Saúde (BR). Protocolo de manejo clínico para o novo coronavírus (2019-nCOV) [Internet]. Brasília: Ministério da Saúde; 2020 [acesso 2020 Fev 18]. 32p. Disponível em: https://portalarquivos2.saude.gov.br/images/ pdf/2020/fevereiro/11/protocolo-manejo-coronavirus.pdf.
6. Sociedade Brasileira de Atendimento Integrado ao Traumatizado. Recomendações SBAIT COVID-19 para profissionais de saúde [Internet]. São Paulo: SBAIT; 2020 [acesso 2020 Mar 25]. Disponível em: http://blog.sbait.org. br/2020/03/25/recomendacoes-sbait-covid-19/.

7. Asociación Española de Cirujanos Documentos de posicionamiento y recomendaciones: Grupo de trabajo Cirugia-AEC-Covid-19 [Internet]. Madri: AEC; 2020 [acesso 2020 Mar. 23]. Disponível em: https://www.aecirujanos.es/ Documentos-de-posicionamiento-y-recomendaciones-de-la-AEC-en-relacioncon-la-cirugia-y-COVID19 es 1 152.html\#!

8. Zheng $\mathrm{MH}$, Boni L, Fingerhut A. Minimally invasive surgery and the novel coronavirus outbreak: lessons learned in China and Italy [Internet]. Ann. Surg. 2020 Mar [acesso 2020 Mar 20]. Disponível em: https://www.facs.org/aboutacs/covid-19/publications. Acesso em: 20 mar. 2020.

9. Pryor A. SAGES recommendations regarding surgical response to COVID-19 CRISIS [Internet]. Los Angeles: SAGE; 2020 [acesso 2020 Mar 19]. Disponível em: https://www.sages.org/recommendations-surgical-response-covid-19/.

10. American College of Surgeons. COVID-19:Guidance for Triageof NonEmergentSurgical Procedures: Emergent General Surgery [Internet]. Chicago: FACS; 2020 [acesso 2020 Mar 24]. Disponível em: https://www.facs.org/ covid-19/clinical-guidance/elective-case.

Como citar este artigo/How to cite this article:

Lima DS, Leite JAD Filho, Gurgel MVSA, Aguiar AF Neto, Costa EFM, Maia FXF Filho, et al. Recomendações para cirurgia de emergência durante a pandemia do COVID-19. J Health Biol Sci. 2020 J; 8(1):1-3. 